\title{
Lung recruitment during general anesthesia
}

David Johnson MD

I NCREASED alveolar to arterial oxygen gradients occur in most patients after the induction of anesthesia. Mild hypoxemia (saturation not below $85 \%$ ) is noted in up to half of patients under general anesthesia and more severe hypoxemia (saturation below $85 \%$ ) in up to $20 \%$. The cause of this hypoxemia is an increase in low ventilation to perfusion areas which can become absolute shunts (i.e., no ventilation but persistent perfusion to collapsed alveoli as airways close). The loss of aerated alveoli (atelectasis) occurs as functional residual capacity is reduced below closing capacity and is a consequence of alveolar oxygen re-absorption, dependent lung compression, and loss of surfactant. Atelectasis under general anesthesia routinely involves 5 to $6 \%$ of the lung predominantly in dependent areas, can increase up to $50 \%$ of the lung in cardiothoracic operations, and can persist for multiple postoperative days. ${ }^{1}$ Atelectasis and airway closure explain as much as $74 \%$ of gas exchange impairment noted during routine general anesthesia as these poorly ventilated regions are relatively overperfused. ${ }^{2}$ Atelectasis may be associated with longer length of hospitals stays, ${ }^{3,4}$ pneumonia, initiation of the inflammatory cascade by activating macrophages, and decrease in surfactant production.

Anesthesiologists can minimize atelectasis using four strategies: 1) ventilation with positive end-expiratory pressure (PEEP); 2) lung volume recruitment; 3 ) maintenance of muscular tone; 4) minimization of pulmonary gas re-absorption. The later technique has led to suggestions that the use of high inspired oxygen prior to induction of anesthesia should be re-evaluated. Preoxygenation with $100 \%$ oxygen has not been uniformly adopted by all practioners. ${ }^{5}$ The use of $100 \%$ oxygen for preoxygenation is based upon the rationale of increasing the safety margin if important hypoxemia develops during the induction of general anesthesia. For example, difficult and prolonged intubations without the ability to hand ventilate may be unexpectedly encountered. Even with preoxygenation, significant hypoxemia can occur in healthy patients prior to resumption of spontaneous ventilation after the use of short-acting muscle relaxants. ${ }^{6}$ Rather than eliminate preoxygentation, the use of lower inspired oxygen $(60 \%)$ may limit the formation of atelectasis. ${ }^{7}$ The risk for significant hypoxemia and consequent need of higher inspired oxygen during induction of anesthesia may have a physiological rationale (decreased functional residual capacity with pregnancy or increase metabolic rate in newborns). In the presence of lung pathology and need for high-inspired oxygen even prior to anesthesia, the use of $100 \%$ oxygen during preoxygenation seems more defensible.

More recently the use of lung recruitment maneuvers during routine anesthesia has been suggested in order to minimize atelectasis and the need for high inspired oxygen. The Canadian Journal of Anesthesia has added to this discussion by publishing such an article by Tusman et al. ${ }^{8}$ The rationale of using recruitment maneuvres early after induction of anesthesia is to forestall the atelectasis that can occur after induction. An atelectatic lung may be re-expanded during anesthesia by applying a vital capacity maneuver or by applying an inflation pressure of $40 \mathrm{~cm} \mathrm{H}_{2} \mathrm{O}$ to the lungs. ${ }^{9}$ Rothen et al. ${ }^{10}$ showed that atelectasis was reduced mostly during the first seven to eight seconds of a vital capacity maneuver with virtually no change after this time. A brief lung recruitment maneuver potentially avoids cardiovascular collapse associated with more prolonged high inspiratory pressure. ${ }^{11}$ The composition of inspiratory gas used in the recruitment maneuver plays an important role in the duration of its effects. Lung re-expansion is more sustained if $40 \%$ oxygen in nitrogen is used. If $100 \%$ oxygen is used after re-expansion, lung collapse recurs within a few minutes. After a recruitment maneuver, the lack of oxygenation improvement during ventilation using low-oxygen gas mixtures suggests that low ventilation-perfusion lung areas are still present. ${ }^{10}$

Most information about lung recruitment maneuvers relates to pressure-limited lung-protective ventilatory strategies in order to prevent atelectasis when

From the Department of Anesthesia, Royal University Hospital, Saskatoon, Saskatchewan, Canada.

Address correspondence to: Dr. David Johnson, Department of Anesthesia, Royal University Hospital, Saskatoon, Saskatchewan S7N 0W8, Canada. Phone: 306-655-1279; Fax: 306-655-1279; E-mail: cujecjohnson@shaw.ca 
small tidal volumes and low to moderate PEEP levels are used. ${ }^{12}$ Bond et al. ${ }^{13}$ showed that oxygenation improved after performing recruitment maneuvers during volume-controlled ventilation using low tidal volume ventilation $\left(7 \mathrm{~mL} \cdot \mathrm{kg}^{-1}\right)$ and zero end-expiratory pressure. Rismensberger et al. ${ }^{14}$ showed that a single recruitment maneuver resulted in better oxygenation at a PEEP level less than the lower inflection point of the pressure-volume curve compared with a PEEP level higher than the lower inflection point of the pressure-volume curve and no recruitment maneuver. The lower inflection "point" of the lung pressure-volume curve is noted when airway opening occurs most frequently - not the inspiratory pressure at which lung unit closure no longer occurs. To maximally open the lung and prevent collapse of unstable lung units, it is necessary to apply inspiratory pressures that produce total lung capacity. ${ }^{15}$ Opening of lung units occurs along the entire length of the inspiratory limb of the pressure-volume curve so that tidal opening and closing of some lung units occur at virtually any tidal volume unless the lungs were fully recruited before initiating ventilatory support. Refractory but potentially recruitable lung units tend to be located in dependent zones and require high and sustained airway pressures to open. The inspired pressures required may substantially exceed those sufficient to achieve total lung capacity in a normal lung. ${ }^{16}$ This specific type of recruitment maneuver - the stepwise application of moderately high airway pressure - is less likely to help either patients with primary (pulmonary, as opposed to extrapulmonary) causes of the acute respiratory distress syndrome, those who are receiving ventilatory support in the prone position, or those with atelectasis due to mechanical forces. ${ }^{17}$ Laboratory studies demonstrate that surfactant depletion and oleic models of lung injury are highly recruitable, whereas lung injury inflicted by pneumonia and purely mechanical forces are not. ${ }^{14}$ Moreover, any improved oxygenation resulting from the recruitment maneuver will be transient unless sufficient PEEP is applied to sustain the benefit.

Recruitment maneuvers are not entirely benign. They may do harm by redirecting blood flow due to high alveolar pressure subsequently interfering with hypoxic pulmonary vasoconstriction. The result is hypoxemia during and after the recruitment maneuver. Hypotension is a risk in patients who are hypovolemic, in those with unusually high "transmission" ratios of lung to chest wall compliance, and in those whose adaptive cardiac reserves are blunted by intrinsic disease or medications. Barotrauma should also be considered whenever high and sustained alveolar pressures are used.
Clinical studies employing recruitment maneuvers have shown atelectasis may be improved in healthy patients. ${ }^{18}$ Routine use has not shown to improve outcomes in at least one patient group ${ }^{19}$ with a high incidence of postoperative atelectasis. A number of technical questions regarding recruitment maneuvers remain unanswered. These include what is the ideal inspiratory pressure to be used, what is the time length for each recruitment maneuver, what is the repetition frequency for multiple recruitment maneuvers, and what preoperative, operative, or postoperative times are most critical in the application of recruitment maneuvers.

The more difficult, unanswered question is demonstrating a causal relationship between preventing atelectasis and improved patient outcome (such as the incidence of postoperative pneumonia, postoperative length of hospital stay, need or duration of mechanical ventilation). Thus, at this time, the risk vs benefit of routine recruitment maneuvers during general anesthesia must be questioned. Similar questions should be asked about more technologically sophisticated techniques of ventilation during anesthesia such as airway pressure release ventilation ${ }^{20}$ which may also prevent atelectasis by avoiding the use of supplemental oxygen and maintenance of spontaneous ventilatory effort. The use of therapy that prevents or improves atelectasis but has not been shown to improve patient outcomes is not restricted to anesthesiologists. These therapies include physiotherapy, intermittent positive pressure breathing, and bronchoscopy. It would seem that, yet again, the therapy has been applied before knowing that the problem is being solved. Until anesthesiologists insist upon patient outcome trials before adopting new techniques and therapies, even when the rationale of these therapies would seem most reasonable, we will continue to be limited to expert opinion.

\section{Le recrutement alvéolaire pendant l'anesthésie générale}

Des gradients alvéolo-artériels d'oxygène accrus sont observés chez la plupart des patients après l'induction de l'anesthésie. Une hypoxémie légère (saturation non inférieure à $85 \%$ ) atteint près de la moitié des patients sous anesthésie générale et une hypoxémie plus sévère 
(saturation inférieure à $85 \%$ ), près de $20 \%$. Cette hypoxémie est causée par une augmentation de l'hypoventilation vers les aires de perfusion, ce qui peut évoluer vers des shunts absolus, c'est-à-dire aucune ventilation mais une perfusion persistante vers les alvéoles collabées au moment de la fermeture des voies aériennes. La perte d'alvéoles aérées (atélectasie) survient avec la réduction de la capacité résiduelle fonctionnelle sous le volume de fermeture et est une conséquence de la réabsorption d'oxygène alvéolaire, de la compression du poumon déclive et de la perte de surfactant. L'atélectasie sous anesthésie générale touche habituellement de 5 à $6 \%$ du poumon, surtout dans les régions déclives, peut affecter jusqu'à $50 \%$ du poumon pendant la chirurgie cardio-thoracique et peut persister pour plusieurs jours postopératoires. ${ }^{1}$ L'atélectasie et la fermeture des voies aériennes peuvent expliquer jusqu'à $74 \%$ de l'altération de l'échange gazeux pendant l'anesthésie générale régulière, car ces régions hypoventilées sont relativement surperfusées. ${ }^{2}$ L'atélectasie peut être associée avec des séjours hospitaliers prolongés, ${ }^{3,4}$ la pneumonie, le début d'une cascade inflammatoire par l'activation des macrophages, et la baisse de production de surfactant.

Les anesthésiologistes peuvent réduire l'atélectasie de quatre façons : 1) la ventilation à pression télé-expiratoire positive (PEEP) ; 2) le recrutement du volume pulmonaire ; 3) le maintien du tonus musculaire ; 4) la réduction de la réabsorption pulmonaire des gaz. Selon cette dernière technique nous pourrions avoir à réévaluer l'administration de fortes concentrations d'oxygène avant l'induction de l'anesthésie. La préoxygénation avec 100 \% d'oxygène n'a pas été uniformément adoptée par les praticiens. ${ }^{5}$ Elle est fondée sur l'idée d'accroître la marge de sécurité au cas où une importante hypoxémie se développerait pendant l'induction de l'anesthésie générale. Par exemple, il est possible que nous soyons subitement confrontés à une intubation difficile sans possibilité de ventiler manuellement. Même avec la préoxygénation, une importante hypoxémie peut survenir chez des patients sains avant le retour de la ventilation spontanée à la suite de l'utilisation de myorelaxants à action brève. ${ }^{6}$ Plutôt que d'éliminer la préoxygénation, l'administration d'oxygène à $60 \%$ pourrait limiter la formation d'atélectasie. 7 Le risque d'une importante hypoxémie et de la nécessité subséquente d'administrer de l'oxygène à forte concentration pendant l'induction de l'anesthésie peut avoir un fondement physiologique. En effet, pensons à la réduction de la capacité résiduelle fonctionnelle pendant la grossesse ou à l'augmentation du rythme métabolique chez les nouveau-nés.
En présence d'une pathologie pulmonaire et de la nécessité de forte concentration d'oxygène inspirée juste avant l'anesthésie, l'usage d'oxygène à 100 \% pendant la préoxygénation semble plus justifiable.

Plus récemment, l'usage régulier de manœuvres de recrutement alvéolaire pendant l'anesthésie a été suggéré pour réduire l'atélectasie et la nécessité d'oxygène inspiré à forte concentration. Le Journal canadien d'anesthésie contribue à cette discussion en publiant l'article de Tusman et coll. ${ }^{8}$ L'idée d'utiliser le recrutement peu après l'induction de l'anesthésie est de prévenir l'atélectasie qui pourrait survenir après l'induction. Un poumon atélectasique peut être redilaté pendant l'anesthésie par l'application d'une manœuvre d'inspiration à capacité vitale ou d'une pression d'inspiration à $40 \mathrm{~cm} \mathrm{H_{2 }}$ O. ${ }^{9}$ Rothen et coll. ${ }^{10}$ ont montré que l'atélectasie a été réduite surtout pendant les sept ou huit premières secondes d'une manœuvre d'inspiration à capacité vitale sans changement significatif après ce temps. Un bref recrutement alvéolaire peut éviter un collapsus cardiovasculaire associé à une pression inspiratoire élevée prolongée. ${ }^{11}$ La composition des gaz inspirés, utilisés pour le recrutement, joue un rôle important dans la durée de leurs effets. La redilatation des poumons dure plus longtemps avec $40 \%$ d'oxygène dans de l'azote. Si on administre $100 \%$ d'oxygène après la réexpansion, l'affaissement du poumon revient en quelques minutes. Après le recrutement, l'absence d'amélioration de l'oxygénation pendant la ventilation avec des mélanges gazeux à faible concentration d'oxygène suppose qu'il $y$ a encore des régions du poumon avec des bas ratios de ventilation/perfusion. ${ }^{10}$

La plupart des informations sur les manœuvres de recrutement alvéolaire ont trait aux stratégies de protection pulmonaire limitées par la pression et visent à prévenir l'atélectasie quand on utilise des petits volumes courants et des niveaux de PEEP de faibles à modérés. ${ }^{12}$ Bond et coll. ${ }^{13}$ ont montré que l'oxygénation s'améliore après le recrutement pendant la ventilation limitée par le volume qui utilisent une ventilation à faible volume courant $\left(7 \mathrm{~mL} \cdot \mathrm{kg}^{-1}\right)$ et aucune pression télé-expiratoire. Rismensberger et coll. ${ }^{14}$ ont montré qu'une seule manœuvre de recrutement permet une meilleure oxygénation à un niveau de PEEP plus bas que le point d'inflexion inférieur de la courbe pression-volume, comparé à un niveau de PEEP plus élevé que ce point de la courbe et sans manœuvre de recrutement. Le «point» d'inflexion inférieur de la courbe de pression-volume pulmonaire est identifié quand l'ouverture des voies aériennes se fait le plus fréquemment - et non lorsque la pression inspiratoire à laquelle la fermeture des unités alvéo- 
laires n'est plus manifeste. Pour ouvrir les poumons au maximum et prévenir l'affaissement d'unités alvéolaires instables, il est nécessaire d'appliquer des pressions inspiratoires qui puissent atteindre une capacité pulmonaire totale. ${ }^{15}$ L'ouverture des unités alvéolaires survient tout au long de la branche inspiratoire de la courbe pression-volume de sorte que l'ouverture et la fermeture cycliques de certaines unités alvéolaires surviennent pour presque tout volume courant à moins que les poumons soient complètement recrutés avant le début de l'assistance ventilatoire. Les unités alvéolaires réfractaires, mais possiblement recrutables, surtout localisées dans les zones déclives, nécessitent des pressions élevées et soutenues pour s'ouvrir. Les pressions inspirées nécessaires peuvent dépasser considérablement celles qui suffisent à atteindre la capacité pulmonaire totale d'un poumon normal. ${ }^{16} \mathrm{Ce}$ type de recrutement spécifique - l'application progressive d'une pression modérément élevée dans les voies respiratoires - n'aidera probablement pas les patients atteints du syndrome de détresse respiratoire aiguë de causes primaires (pulmonaires, opposées à extrapulmonaires), ceux qui reçoivent une assistance respiratoire en décubitus ventral ou ceux qui présentent de l'atélectasie d'origine mécanique. ${ }^{17}$ Des études en laboratoire démontrent que la déplétion du surfactant et les modèles oléiques de lésions pulmonaires sont hautement recrutables, tandis que les lésions pulmonaires causées par une pneumonie et des forces purement mécaniques ne le sont pas. ${ }^{14}$ Qui plus est, toute amélioration de l'oxygénation résultant d'une manœuvre de recrutement sera transitoire à moins qu'une PEEP suffisante soit appliquée pour maintenir les bienfaits.

Le recrutement n'est pas tout à fait inoffensif. Il peut nuire en redirigeant le débit sanguin à cause de la pression alvéolaire élevée qui, subséquemment, interfère avec la vasoconstriction pulmonaire hypoxique. Il en résulte une hypoxémie pendant et après la manœuvre de recrutement. L'hypotension est un risque chez les patients hypovolémiques, chez ceux qui présentent des rapports de «transmission» exceptionnellement élevés de compliance entre le poumon et la paroi thoracique et chez ceux dont les réserves cardiaques adaptatives sont émoussées par une affection intrinsèque ou des médicaments. Un barotrauma doit aussi être envisagé quand on utilise des pressions alvéolaires élevées et soutenues.

Des études cliniques sur le recrutement ont montré que l'atélectasie peut être corrigée chez des patients sains. ${ }^{18}$ On a montré, auprès d'au moins un groupe de patients $^{19}$ qui présentaient une incidence élevée d'atélectasie, que son utilisation régulière n'améliorait pas l'évolution. Un certain nombre de questions techniques demeurent sans réponse. Quelle est, par exemple, la pression inspiratoire idéale à utiliser, combien de temps doit durer une manœuvre de recrutement, quelle doit être la fréquence de répétition de manœuvres multiples et quels moments préopératoires, opératoires ou postopératoires sont les plus critiques dans l'application de manœuvres de recrutement.

La question la plus difficile, toujours sans réponse, concerne la démonstration d'une relation causale entre la prévention de l'atélectasie et l'amélioration de l'état du patient (comme l'incidence de pneumonie postopératoire, la longueur du séjour hospitalier postopératoire, la nécessité et la durée de la ventilation mécanique). À présent il faut donc revoir le rapport risque-avantage des manœuvres régulières de recrutement pendant l'anesthésie générale. Il faut aussi s'interroger sur la ventilation de haute technicité utilisée pendant l'anesthésie comme la ventilation avec dépressurisation expiratoire intermittente ${ }^{20}$ qui peut aussi prévenir l'atélectasie en évitant l'usage d'oxygène supplémentaire et s'interroger sur le maintien d'une ventilation spontanée. Les anesthésiologistes ne sont pas les seuls à utiliser un traitement qui prévient ou réduit l'atélectasie sans améliorer nécessairement l'évolution du patient. Ces traitements comprennent la physiothérapie, la respiration au moyen de respirateurs à pression positive intermittente et la bronchoscopie. Il semble, encore une fois, que la thérapie ait été appliquée avant de savoir si le problème se règle. Aussi longtemps que nous ne mettrons pas l'accent sur des d'essais cliniques avant d'adopter de nouvelles techniques et thérapies, même si la logique de ces thérapies semble raisonnable, nous continuerons d'être limités à une opinion d'expert.

\section{References}

1 Hedenstierna G. Alveolar collapse and closure of airways: regular effects of anaesthesia. Clin Physiol Funct Imaging 2003; 23: 123-9.

2 Hedenstierna G. Airway closure, atelectasis and gas exchange during anaesthesia. Minerva Anestesiol 2002; 68: 332-6.

3 Johnson D, Kelm C, Thomson D, Burbridge B, Mayers I. The effect of physical therapy on respiratory complications following cardiac valve surgery. Chest 1996; 109: 638-44.

4 Johnson D, Kelm C, To T, et al. Postoperative physical therapy after coronary artery bypass surgery. Am J Respir Crit Care Med 1995; 152: 953-8.

5 Heck Z, Stegmann JU, Lorenz C, Heck M, Schlack W. Acceptance of preoxygenation in clinical routine by patients and by anaesthesiologists (German). 
Anasthesiol Intensivmed Notfallmed Schmerzther 2001; 36: 471-5.

6 Hayes AH, Breslin DS, Mirakhur RK, Reid JE, O'Hare $R A$. Frequency of haemoglobin desaturation with the use of succinylcholine during rapid sequence induction of anaesthesia. Acta Anaesthesiol Scand 2001; 45: 746-9.

7 Edmark L, Kostova-Aherdan K, Enlund M, Hedenstierna $G$. Optimal oxygen concentration during induction of general anesthesia. Anesthesiology 2003; 98: 28-33.

8 Tusman G, Bohm SH, Suarez-Sipmann F, Turchetto E. Alveolar recruitment improves ventilatory efficiency of the lungs during anesthesia. Can J Anesth 2004; 51 :723-27.

9 Tusman G, Bohm SH, Vazquez de Anda GF, do Campo $J L$, Lachmann B. "Alveolar recruitment strategy" improves arterial oxygenation during general anaesthesia. Br J Anaesth 1999; 82: 8-13.

10 Rothen HU, Sporre B, Engberg G, Wegenius G, Hogman $M$, Hedenstierna $G$. Influence of gas composition on recurrence of atelectasis after a reexpansion maneuver during general anesthesia. Anesthesiology 1995; 82: 832-42.

11 Lapinsky SE, Aubin M, Mehta S, Boiteau P, Slutsky AS. Safety and efficacy of a sustained inflation for alveolar recruitment in adults with respiratory failure. Intensive Care Med 1999; 25: 1297-301.

12 Amato MB, Barbas CS, Medeiros DM, et al. Effect of a protective-ventilation strategy on mortality in the acute respiratory distress syndrome. N Engl J Med 1998; 338: 347-54.

13 Bond JM, McAloon J, Froese AB. Sustained inflations improve respiratory compliance during high-frequency oscillatory ventilation but not during large tidal volume positive-pressure ventilation in rabbits. Crit Care Med 1994; 22: 1269-77.

14 Rismensberger PC, Cox PN, Frndova H, Bryan AC. The open lung during small tidal volume ventilation: concepts of recruitment and "optimal" positive endexpiratory pressure. Crit Care Med 1999; 27:

1946-52.

15 Ward NS, Lin DY, Nelson DL, et al. Successful determination of lower inflection point and maximal compliance in a population of patients with acute respiratory distress syndrome. Crit Care Med 2002; 30: 963-8.

16 Richard JC, Maggiore SM, Jonson B, Mancebo J, Lemaire F, Brochard $L$. Influence of tidal volume on alveolar recruitment. Respective role of PEEP and a recruitment maneuver. Am J Respir Crit Care Med 2001; 163: 1609-13.

17 Lim CM, Jung H, Koh $\Upsilon$, et al. Effect of alveolar recruitment maneuver in early acute respiratory distress syndrome according to antiderecruitment strategy, etiological category of diffuse lung injury, and body position of the patient. Crit Care Med 2003; 31: 411-8.

18 Tusman G, Bobm SH, Tempra A, et al. Effects of recruitment maneuver on atelectasis in anesthetized children. Anesthesiology 2003; 98: 14-22.

19 Boyle M, Way P, Pinfold M, Lawrence J. Comparison of the lower inflection point on the static total respiratory compliance curve with outcomes in postoperative cardiothoracic patients. Am J Crit Care 2001; 10: 399-407.

20 Hedenstierna G, Lattuada M. Gas exchange in the ventilated patient. Curr Opin Crit Care 2002; 8: 39-44. 